\title{
VALIDITY AND RELIABILITY OF A HANDHELD BLOOD GLUCOSE MONITOR DURING EXERCISE AND AN ORAL GLUCOSE TOLERANCE TEST
}

\author{
Davoncie M. Granderson, Clayton L. Camic, Peter J. Chomentowski, \\ Steven M. Howell, and Emerson Sebastião \\ Human Performance Laboratory, Kinesiology and Physical Education, \\ Northern Illinois University, Illinois, USA
}

Original scientific paper

DOI: $10.26582 / \mathrm{k} .51 .2 .10$

\begin{abstract}
:
The purpose of the present study was to examine the validity and reliability of the handheld Nova Max Plus blood glucose monitor during an oral glucose tolerance test and 60-minute bout of exercise. Thirty subjects (mean age $\pm \mathrm{SD}=22.3 \pm 1.9$ years; body mass $=77.6 \pm 14.2 \mathrm{~kg}$ ) volunteered for an oral glucose tolerance test or a 60 -minute treadmill test. Blood glucose concentrations were measured from the fingertip at six time points during both tests. The reference method of blood glucose analysis was the Yellow Springs Instruments (YSI) 2300. Our results indicated that the blood glucose values provided by the Nova Max Plus were significantly $(p<.05)$ greater than the YSI 2300 at all-time points of the oral glucose tolerance test and treadmill test. In addition, the Nova Max Plus exhibited an overall mean absolute relative deviation $( \pm \mathrm{SD})$ of $9.0( \pm 7.0)$ and did not meet the 95\% accuracy requirements of ISO 15197:2013. The Bland-Altman plot for constant error (YSI 2300 - Nova Max Plus) versus the reference method (YSI 2300) indicated an average negative bias $\left(-8.2 \mathrm{mg} \cdot \mathrm{dL}^{-1}\right)$ that increased $(\mathrm{r}=-0.23)$ at higher blood glucose values. Intra-device reliability analyses for the Nova Max Plus demonstrated the ICC was $\mathrm{R}=0.99$ and $\mathrm{CV}=3.0 \%$, with no mean differences between the test and retest values. These findings suggested that the Nova Max Plus provided highly reliable, yet inaccurate blood glucose values compared to the YSI 2300 during the dynamic conditions associated with an oral glucose tolerance test and exercise.
\end{abstract}

Key words: accuracy, reproducibility, treadmill, physical activity, OGTT, point-of-care

\section{Introduction}

Blood glucose is an important physiological variable in clinical settings and human performance laboratories due to its relationship with various medical conditions and as an indicator of energy availability to metabolically active tissue. For example, the measurement of blood glucose following an overnight fast and oral glucose tolerance test (OGTT) are commonly used as screening and diagnostic techniques for diabetes mellitus (American Diabetes Association, 2018). Specifically, fasting blood glucose values of 100-125 $\mathrm{mg} \cdot \mathrm{dL}^{-1}$ are considered "prediabetic", whereas values $>125 \mathrm{mg} \cdot \mathrm{dL}^{-1}$ are classified as "diabetic" (American Diabetes Association, 2018). During the OGTT procedure, blood glucose levels at the two-hour time point of $140-199 \mathrm{mg} \cdot \mathrm{dL}^{-1}$ and $\geq 200 \mathrm{mg} \cdot \mathrm{dL}^{-1}$ are classified as "prediabetic" and "diabetic", respectively (American Diabetes Association, 2018). Glucose levels in the blood can also be monitored during longer bouts of exercise to provide information related to carbohydrate metab- olism and as a preventative measure against low blood sugar (i.e. hypoglycemia). The maintenance of adequate blood glucose is vital for proper functioning of various metabolic processes and reflects the balance of carbohydrate intake, cellular uptake, and glucose release by the liver (Coggan, 1997; Suh, et al., 2007). Although exercise provides dynamic conditions in which glucose uptake by active skeletal muscles increases, this is balanced by glucose release from the liver, thereby maintaining nearly constant blood glucose values that usually change by less than 5-10 $\mathrm{mg}^{\circ} \mathrm{dL}^{-1}$ (Suh, Paik, \& Jacobs, 2007; Zinker, Britz, \& Brooks, 1990). In addition, the use of physical activity as a powerful preventative and therapeutic agent against glucose-related disorders has been well-documented (Jelleyman, et al., 2017). Among other benefits, physical activity stimulates the cellular uptake of glucose independent of insulin, promotes insulin sensitivity, weight loss, and favorable blood glucose levels (Boulé, Kenny, Haddad, Wells, \& Sigal, 2003; Boulé, et al., 2005; Jelleyman, et al., 2017). Hypoglycemia, 
or low blood sugar, is a state in which the energy demands of the body may not be supplied, thereby leading to fatigue, decrements in mental acuity, and unconsciousness in extreme cases (American Diabetes Association, 2018; Cryer, 2007). The risk of hypoglycemia is obviously of greatest concern to diabetics, but can also play a factor in athletic performance during intense or extensive exercise and be indicative of fatigue-inducing glycogen depletion (Cermak \& van Loon, 2013). Thus, the measurement of blood glucose is critical for the health, safety, and physical performance of both the clinical and athletic individuals in home-, clinical-, or laboratory-based environments.

The reference techniques for measuring blood glucose typically involve using enzymatic assays or isotope dilution gas chromatography-mass spectrometry (Hagvik, 2007). For example, the YSI 2300 STAT PLUS (Yellow Springs Instruments, Ohio) analyzes blood glucose using the glucose oxidase method and is considered the reference method by the U.S. Food and Drug Administration that most other glucose meters are calibrated against (Ekhlaspour, et al., 2017). Although the YSI 2300 STAT PLUS offers a high level of precision $\left( \pm 2 \%\right.$ of the reading or $2.5 \mathrm{mg} \cdot \mathrm{dL}^{-1}$ ) (YSI Life Sciences, 2009), it requires expensive and timeconsuming operation and maintenance procedures that are only realistic in laboratory and clinical settings with trained personnel. In addition, the YSI 2300 is designed to be stationary and requires an external energy source. Thus, for quick and easy blood glucose measurement in the field (e.g. health fairs, schools, exercise facilities), or as a point-of-care test at home, the portable handheld glucose monitors provide an attractive alternate over the more complex, laboratory-based procedures. These portable handheld glucose monitors are considered highly user-friendly (e.g. simple to operate, small size, battery-powered), cost effective, and can produce results in approximately five seconds from less than a drop of blood (i.e. $0.3 \mu \mathrm{L}$ ). Although measures of quality control have been assessed in many handheld glucose monitors in the fasted state (Robinson \& Sharp, 2012; Tack, et al., 2012; Thomas, et al., 2008) or in vitro (Bedini, Wallace, Pardo, \& Petruschke, 2015; Ekhlaspour, et al., 2017), there are limited data regarding these factors in vivo under dynamic conditions of blood glucose. Therefore, the purpose of the present study was to examine the validity and reliability of the handheld Nova Max Plus (Nova Biomedical Corp., Waltham, MA) blood glucose monitor during an OGTT and 60-minute bout of exercise.

\section{Methods}

\section{Subjects}

A total of 30 subjects (mean age $\pm \mathrm{SD}=22.3 \pm 1.9$ years; body mass $=77.6 \pm 14.2 \mathrm{~kg}$; body height $=$

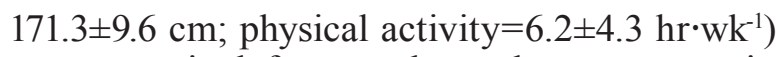
were recruited from undergraduate courses in kinesiology and physical education to participate in a single visit to the Human Performance Laboratory for an OGTT $(n=15)$ or a 60 -minute treadmill exercise test $(\mathrm{n}=15)$. The subjects did not report or exhibit any of the following that could significantly affect the outcome of the study: (i) history of medical or surgical events, including cardiovascular disease, heart disease, hypertension, diabetes mellitus, insulin resistance, hypoglycemia, or any other metabolic, renal, hepatic, or musculoskeletal disorder; (ii) phobia to needles or finger-stick; (iii) fasting blood glucose level $>100 \mathrm{mg} \cdot \mathrm{dL}^{-1}$, or (iv) any current physical injury due to the physical demands and requirements of the study. Subjects recruited for the OGTT $(n=15)$ were asked to avoid eating or drinking anything other than water for eight hours prior to their visit, whereas the subjects recruited to participate in the 60-minute treadmill exercise $(n=15)$ were asked to avoid eating or drinking anything other than water for 2-3 hours prior to their visit. The study was approved by the Institutional Review Board at Northern Illinois University, and all participants completed a health history questionnaire and signed a written informed consent document prior to testing.

\section{Oral Glucose Tolerance Test (OGTT)}

Fifteen subjects (mean age $\pm \mathrm{SD}=22.4 \pm 1.7$ years; body mass $=77.0 \pm 13.2 \mathrm{~kg}$; body height $=170.5 \pm 10.6$ $\mathrm{cm}$; physical activity $=5.0 \pm 2.5 \mathrm{hr} \cdot \mathrm{wk}^{-1}$ ) were tested in the morning following an 8-hour overnight fast. Subjects had a baseline ( $0 \mathrm{~min})$ blood glucose measurement taken immediately prior to the ingestion of the glucose drink to ensure the subjects were in a fasted state with normal levels of blood glucose $\left(<100 \mathrm{mg} \cdot \mathrm{dL}^{-1}\right)$. If blood glucose levels were within normal range, the subjects were asked to ingest a drink $(296 \mathrm{~mL})$ that consisted of 75 grams of glucose (ThermoFisher Scientific, Waltham, MA). Subjects were then asked to sit quietly in the laboratory with their blood glucose being measured at the 10-, 20-, $30-, 60-$, and 90-minute time points of the OGTT.

\section{Treadmill exercise}

Fifteen subjects (mean age $\pm \mathrm{SD}=22.3 \pm 2.2$ years; body mass $=78.2 \pm 15.6 \mathrm{~kg}$; body height $=172.2 \pm 8.7$ $\mathrm{cm}$; physical activity $=7.3 \pm 5.4 \mathrm{hr} \cdot \mathrm{wk}^{-1}$ ) were tested for their blood glucose responses during 60 minutes of walking on a treadmill (Woodway Desmo HP, Waukesha, WI). Subjects had a baseline (0 min) blood glucose measurement taken immediately prior to the treadmill test in which they were asked to walk at $5.6 \mathrm{~km} \cdot \mathrm{hr}^{-1}$ for a total of 60 minutes. This velocity was selected to exceed the minimum walking velocity $\left(4.8 \mathrm{~km} \cdot \mathrm{hr}^{-1}\right)$ recommended for health benefits (Kelly, Murphy, Oja, Murtagh, \& Foster, 2011). Blood glucose was measured at the 
5-, 10-, 15-, 30-, and 60-minute time points of the test. At each of these time points, the treadmill belt was briefly paused to safely allow for the measurement of blood glucose with the subject standing still. Following each measurement, the subject was instructed to continue the test at $5.6 \mathrm{~km} \cdot \mathrm{hr}^{-1}$ until the 60 -minute time point had been reached.

\section{Measurement of blood glucose}

Blood glucose concentrations were measured from the fingertip at six different time points during the $\operatorname{OGTT}(0,10,20,30,60$, and $90 \mathrm{~min})$ and treadmill test $(0,5,10,15,30$, and $60 \mathrm{~min})$. Following the standard procedure, the finger was cleaned with alcohol before the initial finger prick and prior to each blood sample. In addition, the initial blood drop that formed on the finger was wiped away and the subsequent blood drops were sampled for analysis and used as the representative blood glucose concentrations. During the OGTTs and treadmill tests, one blood sample was analyzed four times at each time point, two by the YSI 2300 and two by the handheld device (Nova Max Plus, Nova Biomedical Corp., Waltham, MA). Specifically, $50 \mu \mathrm{L}$ of whole capillary blood was collected at each time point using two heparinized capillary tubes and transferred into a $1.5 \mathrm{~mL}$ graduated natural micro-centrifuge tube. This sample was analyzed twice by the YSI 2300 and twice by the Nova Max Plus using a micropipette to present the sample to the glucose monitor. The criterion reference method of blood glucose analysis used in the present study was the Yellow Springs Instruments (YSI) 2300 Stat Plus Glucose and Lactate analyzer (Yellow Springs, $\mathrm{OH})$. The YSI 2300 measures blood glucose from whole blood using the glucose oxidase method. This technique required two, $25 \mu \mathrm{L}$ of whole blood collected into heparinized capillary tubes from a finger-stick. Duplicate YSI blood glucose results were required to be within $\pm 4 \mathrm{mg} \cdot \mathrm{dL}^{-1}$ to be used for analysis and the average of these two measurements was used as the representative YSI 2300 value. The YSI 2300 whole blood glucose values were then converted to plasma equivalents (whole blood $* 1.12=$ plasma), and this value was compared with the test strip results of the handheld device (Nova Max Plus). For quality control, the YSI 2300 was calibrated against 180 and $900 \mathrm{mg} \cdot \mathrm{dL}^{-1}$ solutions according to the manufacturer guidelines (YSI Life Sciences, 2009). The handheld device required $0.3 \mu \mathrm{L}$ of whole blood for the measurement of blood glucose within $20-600 \mathrm{mg} \cdot \mathrm{dL}^{-1}$ using an electrochemical glucose oxidase biosensor. In addition, the handheld device was checked for accuracy against a known quality control solution prior to testing as recommended by the manufacturer (Nova Biomedical Corp.).

\section{Data analysis}

Data were analyzed using SPSS version 24.0 (SPSS Inc. Armonk, NY: IBM Corp.) and provided as mean $\pm \mathrm{SD}$. Accuracy of the handheld glucose monitor compared to the reference YSI 2300 values involved multiple analyses. First, mean differences in blood glucose values between the YSI 2300 and handheld monitor across time during the OGTT and exercise tests were assessed using separate two-way (method $\mathrm{x}$ time) analyses of variance (ANOVAs) with repeated measures and followup paired-samples $t$-tests when appropriate. Accuracy was also assessed through: 1) the calculation of the mean absolute relative difference (MARD); 2) comparing test results of the handheld monitor against the International Organization for Standardization performance standards (ISO 15197:2013); and 3) Bland-Altman (1986) plots with associated calculation of constant error $(\mathrm{CE}=$ mean difference for YSI blood glucose - handheld blood glucose) and Pearson correlation coefficient (r). The MARD was calculated as the mean of individual absolute relative difference (ARD) values. Each individual ARD was calculated as:

$\mathrm{ARD}=100 \frac{(\text { Nova Max value-YSI } 2300 \text { value })}{\text { YSI } 2300 \text { value }}$

Intra-device reliability for the handheld glucose meter was assessed using coefficient of variation (CV), intra-class correlation coefficient (ICC), and paired-samples $t$-tests. A p-value of 0.05 was used for statistical significance.

\section{Results}

Accuracy. The results of the two-way repeated measures ANOVA for blood glucose values during the OGTT indicated there was a significant $(\mathrm{p}<.05)$ method $\mathrm{x}$ time interaction $[F(5,70)=2.806, \mathrm{p}=.023]$. Follow-up paired-samples $t$-tests indicated that the handheld monitor resulted in significantly greater blood glucose values than the YSI 2300 at all time points (Table 1). For blood glucose values during the treadmill exercise test, there was no significant method $\mathrm{x}$ time interaction $[F(5,70)=0.868, \mathrm{p}=.507]$ or main effect for time $[F(5,70)=0.533, \mathrm{p}=.751]$, but there was a main effect for method $[F(1,14)=12.191$, $\mathrm{p}=.004]$. A follow-up paired samples $t$-test indicated that the handheld monitor $\left(86.2 \pm 18.8 \mathrm{mg} \cdot \mathrm{dL}^{-1}\right)$ resulted in significantly greater blood glucose values than the YSI $2300\left(81.3 \pm 19.8 \mathrm{mg}^{\cdot} \mathrm{dL}^{-1}\right)$ (collapsed across time) (Table 1). The MARD $( \pm \mathrm{SD})$ and accuracy values for the Nova Max Plus compared to the reference YSI 2300 method are provided in Table 2. Specifically, the overall MARD $\pm \mathrm{SD}$ was $9.0 \pm 7.0 \%$ and the combined blood glucose values (OGTT and treadmill exercise tests, $n=180$ ) that were within the 
Table 1. Mean \pm SD blood glucose values from the YSI 2300 and Nova Max Plus during an oral glucose tolerance test and treadmill exercise

\begin{tabular}{|c|c|c|c|c|c|c|}
\hline \multicolumn{7}{|c|}{ Oral glucose tolerance test time (minutes) $(n=15)$} \\
\hline Method & 0 & 10 & 20 & 30 & 60 & 90 \\
\hline YSI $2300\left(\mathrm{mg} \cdot \mathrm{dL}^{-1}\right)$ & $87.3 \pm 4.7$ & $116.0 \pm 10.4$ & $143.4 \pm 13.5$ & $158.5 \pm 16.6$ & $132.7 \pm 21.8$ & $113.5 \pm 12.7$ \\
\hline Nova Max Plus (mg $\left.\cdot \mathrm{dL}^{-1}\right)$ & $97.3 \pm 8.0^{*}$ & $130.9 \pm 12.6^{*}$ & $157.7 \pm 17.4^{*}$ & $170.9 \pm 19.7^{*}$ & $141.5 \pm 22.3^{*}$ & $120.2 \pm 15.2^{*}$ \\
\hline \multicolumn{7}{|c|}{ Treadmill exercise time (minutes) $(n=15)$} \\
\hline Method & 0 & 5 & 10 & 15 & 30 & 60 \\
\hline YSI $2300\left(\mathrm{mg} \cdot \mathrm{dL}^{-1}\right)$ & $86.1 \pm 9.9$ & $84.6 \pm 9.5$ & $85.9 \pm 8.2$ & $85.3 \pm 7.2$ & $86.1 \pm 5.4$ & $87.6 \pm 6.0$ \\
\hline Nova Max Plus $\left(\mathrm{mg} \cdot \mathrm{dL}^{-1}\right) \dagger$ & $91.8 \pm 9.2$ & $90.6 \pm 8.1$ & $89.9 \pm 8.0$ & $90.7 \pm 8.5$ & $92.4 \pm 8.0$ & $90.7 \pm 7.5$ \\
\hline
\end{tabular}

Note. *Significantly $(p<.05)$ greater than YSI 2300 blood glucose value. † Main effect $(p<.05)$ for method (Nova Max Plus $>$ YSI 2300) collapsed across time.

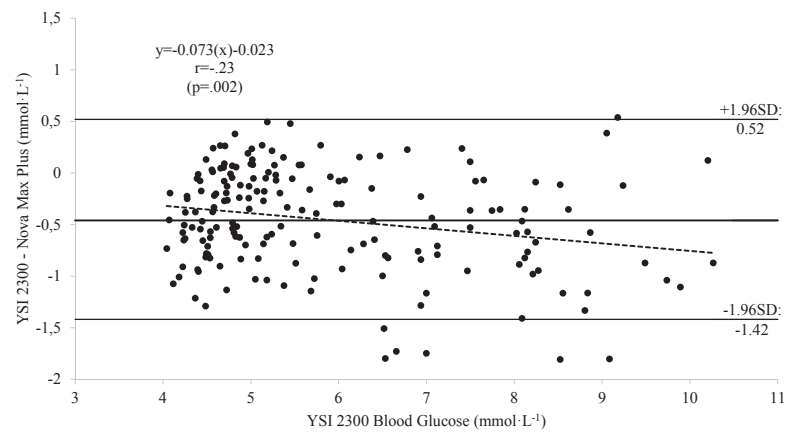

Figure 1. The relationship between constant error (YSI 2300 - Nova Max Plus) and the YSI 2300 blood glucose reference values $(n=180 ; 30$ subjects $x 6$ time points $=180)$. A dashed line represents the regression line.

ISO 15197:2013 criteria was 87.2\% (157/180) (Table $2)$. The Bland-Altman plot indicated there was a significant $(\mathrm{p}<.05)$ negative relationship $(\mathrm{r}=-0.23)$ for CE (YSI 2300 - Nova Max Plus) versus the reference method (YSI 2300) as well as a negative bias $\left(\mathrm{CE}=-8.15 \mathrm{mg}^{\circ} \mathrm{dL}^{-1}\right)$ (Figure 1).

Reliability. The test-retest intra-device reliability analyses for the Nova Max Plus during the OGTT and treadmill exercise test indicated there was no significant ( $\mathrm{p}>.05)$ mean difference between measurement $1\left(113.9 \pm 31.2 \mathrm{mg} \cdot \mathrm{dL}^{-1}\right)$ and measurement $2\left(113.6 \pm 31.4 \mathrm{mg} \cdot \mathrm{dL}^{-1}\right)$ on the combined data $(\mathrm{n}=180)$. The ICC and CV associated with measurement 1 versus measurement 2 were $R=0.99$ and $3.0 \%$, respectively. In addition, the relationship between measurement 1 and measurement 2 from the Nova Max Plus resulted in a significant correlation $(\mathrm{r}=0.99)$ (Figure 2).

\section{Discussion and conclusions}

This study examined the validity and reliability of the handheld Nova Max Plus (Nova Biomedical Corp., Waltham, MA) blood glucose monitor during an OGTT and 60-minute bout of exercise. The blood glucose responses during the OGTT and 60 minutes of treadmill exercise (Table 1) were comparable to those previously reported for healthy individuals (Liu, et al. 2008; Zinker, et al. 1990). Specifically,

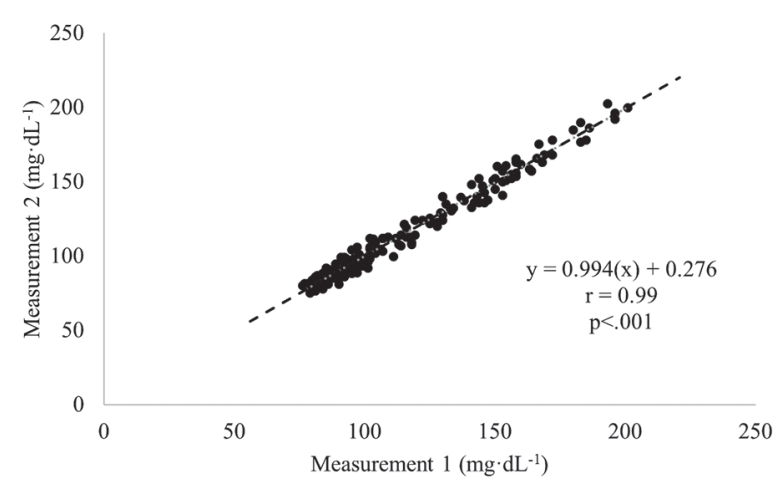

Figure 2. Test-retest reliability for the Nova Max Plus $(n=$ 180; 30 subjects $x 6$ time points $=180$ ).

the blood glucose values (mean \pm SD) during the 75 -g OGTT at the 0 -min $\left(87.3 \pm 4.7 \mathrm{mg} \cdot \mathrm{dL}^{-1}\right)$ and 90 -min $\left(113.5 \pm 12.7 \mathrm{mg}^{\circ} \mathrm{dL}^{-1}\right)$ time points were below those associated with prediabetes (fasting: 100-125

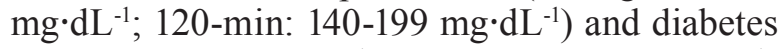
(fasting: $>125 \mathrm{mg} \cdot \mathrm{dL}^{-1} ; 120-\mathrm{min}:>200 \mathrm{mg} \cdot \mathrm{dL}^{-1}$ ) (American Diabetes Association, 2018). In addition, the range in blood glucose values $(84.6 \pm 9.5$ to $87.6 \pm 6.0 \mathrm{mg} \cdot \mathrm{dL}^{-1}$ ) during the treadmill test were similar to those reported by Zinker et al. (1990) over 60 minutes of moderate intensity exercise. Thus, the subjects in the present study exhibited blood glucose responses during the OGTT and 60-min exercise test that were consistent with healthy, nondiabetic individuals of comparable age (Liu, et al., 2008; Zinker, et al., 1990).

Accuracy. One of the main findings of the present study was that the Nova Max Plus provided significantly greater blood glucose values than the reference method (YSI 2300) under both conditions at all time points of the tests (Table 1). Therefore, the Nova Max Plus consistently overestimated blood glucose provided by the YSI 2300. In addition, the MARD and ISO 15197:2013 requirements were used to examine the accuracy of the handheld monitor within different reference ranges of blood glucose. Specifically, the calculation of MARD provides the mean absolute difference 
value between the Nova Max Plus and reference method (YSI 2300) expressed as a percent of the reference value (Obermaier, et al. 2013). Thus, the MARD describes the magnitude of percentage bias of each measurement (Tack, et al. 2012) and a lower MARD value is associated with higher accuracy (i.e. closer to the reference value). Although there are no set criteria for an "accurate" versus "inaccurate" MARD, values ranging from 4.2 to 39.2 have been reported for multiple handheld units (Ekhlaspour, et al. 2017; Robinson \& Sharp, 2012; Tack, et al. 2012). Our results indicated that the Nova Max Plus exhibited an overall MARD $( \pm S D)$ of $9.0( \pm 7.0)(\mathrm{n}=180)$, and values of $9.0( \pm 7.3)$ and $9.1( \pm 6.6)$ at the low $\left(<100 \mathrm{mg} \cdot \mathrm{dL}^{-1}, \mathrm{n}=105\right)$ and high $\left(>100 \mathrm{mg} \cdot \mathrm{dL}^{-1}, \mathrm{n}=75\right)$ reference ranges of blood glucose, respectively (Table 2). The overall MARD value of 9.0 was consistent with previous findings for the Nova Max Plus under fasting conditions (MARD = 8.1) (Robinson \& Sharp, 2012) and in vitro preparations $(\mathrm{MARD}=9.7)($ Ekhlaspour, et al. 2017) that involved blood samples modified for glucose $\left(20-440 \mathrm{mg} \cdot \mathrm{dL}^{-1}\right)$. In addition, Ekhlaspour et al. (2017) reported overall MARD $( \pm \mathrm{SD})$ values ranging from $5.6( \pm 6.4)$ to $20.8( \pm 16.6)$ for 17 different handheld blood glucose monitors versus the YSI 2300, with the Nova Max Plus ranking $8^{\text {th }}$ for the lowest MARD (9.7). Therefore, the findings of the present study and those of others (Ekhlaspour, et al. 2017; Robinson \& Sharp, 2012) indicated that Nova Max Plus provides consistent MARD values (9.0-9.7) within a wide range of blood glucose during fasting and dynamic conditions.

Although the present study was not conducted in vitro according to the strict compliance guidelines of ISO 15197:2013 that require the manipulation of blood glucose and hematocrit, their accuracy criteria was utilized in the evaluation of validity. The ISO 15197:2013 accuracy requirements for blood glucose monitoring systems specify that $95 \%$ of the measured values be within $\pm 15 \mathrm{mg} \cdot \mathrm{dL}^{-1}$ of the reference for values $<100 \mathrm{mg} \cdot \mathrm{dL}^{-1}$ and within $\pm 15 \%$ for values $>100 \mathrm{mg} \mathrm{dL}^{-1}$ (ISO 15197:2013). Overall, the Nova Max Plus did not meet the 95\% accuracy criteria, yielding only $87.2 \%$ of readings within the required range (Table 2). To further examine the accuracy of the Nova Max Plus, we determined the percentage of readings that fell within the required range for values above and below $100 \mathrm{mg} \cdot \mathrm{dL}^{-1}$. In the reference range $<100 \mathrm{mg} \cdot \mathrm{dL}^{-1}$, the Nova Max Plus was only within $\pm 15 \mathrm{mg} \cdot \mathrm{dL}^{-1}$ for $87.6 \%(92 / 105)$ of the values. For the reference range $>100 \mathrm{mg} \cdot \mathrm{dL}^{-1}$, only $86.7 \%(65 / 75)$ of the values were within the ISO 15197:2013 requirements of $\pm 15 \%$. The results of these analyses indicated that the Nova Max Plus did not meet the ISO requirements in any of the three ranges (high, low, overall) of blood glucose that we examined. These findings are consistent with previous studies that have examined Nova Max Plus within fasting individuals and in vitro (Ekhlaspour, et al., 2017; Robinson \& Sharp, 2012) in which the Nova Max was found to have overall ISO 15197:2013 accuracy values of $89.0 \%$ and $88.2 \%$, respectively.

The relationship between CE (YSI 2300 - Nova Max Plus) and the reference (YSI 2300) blood glucose values was analyzed using the method of Bland and Altman (1986) (Figure 1). For this relationship, the mean CE was $-8.15 \mathrm{mg} \cdot \mathrm{dL}^{-1}$ and correlation coefficient was $r=-0.23$. Constant error $(\mathrm{CE})$ provides the mean difference between the actual (YSI 2300) and predicted (Nova Max Plus) blood glucose values. Specifically, the negative CE of $-8.15 \mathrm{mg} \cdot \mathrm{dL}^{-1}$ indicated that, on average, the Nova Max Plus overestimated the measurement of blood glucose by $8.15 \mathrm{mg} \cdot \mathrm{dL}^{-1}$. In fact, $81 \%(145 / 180)$ of the blood glucose measurements from the Nova Max Plus resulted in overestimated values. The negative correlation $(r=-0.23)$ suggested that the absolute $C E$ values became greater at the high end of the reference (YSI 2300) blood glucose values. Thus, the Nova Max Plus overestimated blood glucose values by approximately $8.15 \mathrm{mg} \cdot \mathrm{dL}^{-1}$ and this error tended to increase at higher blood glucose values.

Reliability. The intra-device test-retest reliability analyses of the Nova Max Plus were performed

Table 2. Accuracy of Nova Max Plus in different reference blood glucose ranges

\begin{tabular}{|c|c|c|c|}
\hline & Overall & $<100 \mathrm{mg} \cdot \mathrm{dL}^{-1}$ & $>100 \mathrm{mg} \cdot \mathrm{dL}^{-1}$ \\
\hline MARD $\pm S D(\%)$ & $9.0 \pm 7.0$ & $9.0 \pm 7.3$ & $9.1 \pm 6.6$ \\
\hline $95 \% \mathrm{Cl}$ & 8.0 to 10.1 & 7.6 to 10.4 & 7.6 to 10.6 \\
\hline \multicolumn{4}{|l|}{ Values within } \\
\hline $\pm 5 \% / 5 \mathrm{mg} \cdot \mathrm{dL}^{-1}$ & $80 / 180(44.4 \%)$ & $53 / 105$ (51.5\%) & $27 / 75(36.0 \%)$ \\
\hline $\pm 10 \% / 10 \mathrm{mg} \cdot \mathrm{dL}^{-1}$ & $149 / 180(82.8 \%)$ & $76 / 105$ (72.4\%) & $43 / 75(57.3 \%)$ \\
\hline $\pm 15 \% / 15 \mathrm{mg} \cdot \mathrm{dL}^{-1}$ & $157 / 180(87.2 \%)$ & $92 / 105$ (87.6\%) & $65 / 75(86.7 \%)$ \\
\hline ISO standards* met? & No & No & No \\
\hline
\end{tabular}

Note. *ISO $15197: 2013$ standards require that $95 \%$ of values $<100 \mathrm{mg} \cdot \mathrm{dL}^{-1}$ be within $\pm 15 \mathrm{mg} \cdot \mathrm{dL}^{-1}$ of reference value and $95 \%$ of values $>100 \mathrm{mg} \cdot \mathrm{dL}^{-1}$ be within $\pm 15 \%$. 
on the two blood glucose measurements taken at each time point during the OGTT and treadmill test (30 subjects x 6 time points $=180$ comparisons). As recommended by Atkinson and Nevill (1998), a number of statistical methods should be utilized for assessing reliability in variables relevant to sports medicine to provide a comprehensive analysis. Our findings indicated there were no significant mean differences between the first $\left(113.9 \pm 31.2 \mathrm{mg} \cdot \mathrm{dL}^{-1}\right)$ and second $\left(113.6 \pm 31.4 \mathrm{mg} \cdot \mathrm{dL}^{-1}\right)$ measurements. Although suitable, the $t$-statistics does not provide an indication of random variation between tests and should be interpreted with caution (Atkinson \& Nevill, 1998). Thus, we also utilized the ICC and $\mathrm{CV}$ as two additional statistical procedures that are commonly used to assess relative and absolute reliability, respectively (McLain, et al., 2015). The ICC is used to describe the strength of the similarity of the values within a group and categories suggested by Vincent and Weir (2012) include "excellent" ( $R$ close to 1$)$, "high" $(R>0.90)$, "good" $(R=0.80$ $0.89)$, and "questionable" $(R=0.70-0.79)$. For $\mathrm{CV}$, an acceptable boundary of $<10 \%$ has been previously proposed (Atkinson \& Nevill, 1998; McLain, et al. 2015). Therefore, the Nova Max Plus exhibited excellent relative reliability (ICC, $R=0.99$ ) and acceptable absolute reliability ( $\mathrm{CV}=3.0 \%)$. Collectively, these analyses indicated that the Nova Max Plus provided highly reliable blood glucose values on two consecutive measurements taken at the same time points during the OGTT and treadmill test.

Implications and limitations. The present study provides meaningful data for health care providers, clinicians, coaches and trainers in human performance, and other fitness professionals for assessing blood glucose in the field or laboratory during exercise, fasting, or postprandial. Overall, our findings suggested that readings from the Nova Max Plus for the screening or diagnosis of blood glucose-related disorders at health fairs, schools, in the laboratory, etc., should be interpreted with caution. These findings should also be taken into consideration for individuals that require accurate blood glucose readings that determine the appropriateness for exercise, food ingestion, or insulin injections. It should be noted, however, that this study was not without limitations. Specifically, our sample only included healthy subjects without any glucose-related disorders. It is likely that many individuals that use Nova Max Plus handheld glucose monitors suffer from insulin resistance, diabetes, or other metabolic problems. In addition, the blood glucose responses to the OGTT in our sample of healthy individuals did not exceed $200 \mathrm{mg} \cdot \mathrm{dL}^{-1}$. Therefore, the validity and reliability of the Nova Max Plus were not assessed using a blood glucose

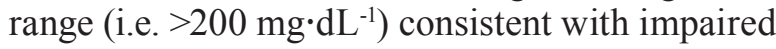
glucose homeostasis. Importantly, however, our findings indicated that higher blood glucose values were associated with less accurate readings from the Nova Max Plus. Future studies should consider examining the accuracy and reliability of the Nova Max Plus and other handheld blood glucose monitors during more intense and longer duration bouts of exercise that may be associated with hypoglycemia or glycogen depletion in athletes, or during dynamic conditions in clinical populations (i.e. Type 1 and 2 diabetes) that exhibit large ranges in blood glucose responses.

In summary, the blood glucose values provided by the handheld Nova Max Plus were significantly higher than those of the YSI 2300 reference method at all time points of the OGTT and 60-minute treadmill test. In addition, the Nova Max Plus exhibited an overall MARD $( \pm \mathrm{SD})$ of $9.0( \pm 7.0)$ and failed to meet the $95 \%$ accuracy requirements of ISO $15197: 2013$ (only $87.2 \%$ of all values met the criteria). The Bland-Altman plot for CE (YSI 2300 - Nova Max Plus) versus the reference method (YSI $2300)$ indicated an average negative bias $(\mathrm{CE}=-8.2$ $\left.\mathrm{mg} \cdot \mathrm{dL}^{-1}\right)$ that tended to increase $(\mathrm{r}=-0.23)$ at higher blood glucose values. The intra-device reliability analyses of the Nova Max Plus, however, demonstrated that the ICC was $R=0.99$ and $\mathrm{CV}=3.0 \%$, with no significant mean differences between the test and retest values. These findings suggested that the Nova Max Plus provided highly reliable, yet inaccurate blood glucose values compared to the YSI 2300 during the dynamic conditions associated with an OGTT and exercise.

\section{References}

American Diabetes Association. (2018). 2. Classification and diagnosis of diabetes: Standards of medical care in diabetes-2018. Diabetes Care, 41(Supplement 1), S13-S27. doi: 10.2337/dc18-S002

Atkinson, G., \& Nevill, A.M. (1998) Statistical methods for assessing measurement error (reliability) in variables relevant to sports medicine. Sports Medicine, 26(4), 217-238.

Bedini, J.L., Wallace, J.F., Pardo, S., \& Petruschke, T. (2015). Performance evaluation of three blood glucose monitoring systems using ISO 15197: 2013 accuracy criteria, consensus and surveillance error grid analyses, and insulin dosing error modeling in a hospital setting. Journal of Diabetes Science and Technology, 10(1), 85-92. doi: $10.1177 / 1932296815609368$

Bland, J.M., \& Altman, D.G. (1986). Statistical methods for assessing agreement between two methods of clinical measurement. The Lancet, 327(8476), 307-310. 
Boulé, N.G., Kenny, G.P., Haddad, E., Wells, G.A., \& Sigal, R.J. (2003). Meta-analysis of the effect of structured exercise training on cardiorespiratory fitness in Type 2 diabetes mellitus. Diabetologia, 46(8), 1071-1081. doi: $10.1007 / \mathrm{s} 00125-003-1160-2$

Boulé, N.G., Weisnagel, S.J., Lakka, T.A., Tremblay, A., Bergman, R.N., Rankinen, T., et al. (2005). Effects of exercise training on glucose homeostasis: The HERITAGE family study. Diabetes Care, 28(1), 108-114.

Cermak, N.M., \& van Loon, L.J. (2013). The use of carbohydrates during exercise as an ergogenic aid. Sports Medicine, 43(11), 1139-1155. doi: 10.1007/s40279-013-0079-0

Coggan, A.R. (1997). Plasma glucose metabolism during exercise: Effect of endurance training in humans. Medicine and Science in Sports and Exercise, 29(5), 620-627.

Cryer, P.E. (2007). Hypoglycemia, functional brain failure, and brain death. The Journal of Clinical Investigation, 117(4), 868-870. doi: 10.1172/JCI31669

Ekhlaspour, L., Mondesir, D., Lautsch, N., Balliro, C., Hillard, M., Magyar, K., et al. (2017). Comparative accuracy of 17 point-of-care glucose meters. Journal of Diabetes and Technology, 11(3), 558-566. doi: 10.1177/1932296816672237

Hagvik, J. (2007). Glucose measurement: Time for a gold standard. Journal of Diabetes Science and Technology, 1(2), 169-172. doi: 10.1177/193229680700100205

International Organization for Standardization. (2013). ISO 15197:2013: In vitro diagnostic test systems - Requirements for blood-glucose monitoring systems for self-testing in managing diabetes mellitus. Retrieved January 4, 2018 from: https://www.glucomen.co.uk/wp-content/themes/glucomen/assets/pdfs/Areo_ISO_Accuracy_bro.pdf

Jelleyman, C., Edwardson, C.L., Henson, J., Gray, L.J., Rowlands, A.V., Khunti, K., et al. (2017). Associations of physical activity intensities with markers of insulin sensitivity. Medicine and Science in Sports and Exercise, 49(12), 2451-2458. doi: 10.1249/MSS.0000000000001381

Kelly, P., Murphy, M., Oja, P., Murtagh, E.M., \& Foster, C. (2011). Estimates of number of people in England who attain or exceed vigorous intensity exercise by walking at $3 \mathrm{mph}$. Journal of Sports Sciences, 29(15), 1629-1634. doi: 10.1080/02640414.2011.609897

Liu, T., Liu, Y., Lee, S., Huang, C., Chien, K., Cheng, I., et al. (2008). Effects of short-term detraining on measures of obesity and glucose tolerance in elite athletes. Journal of Sports Sciences, 26(9), 919-925. doi: 10.1080/02640410801885925

McLain, T.A., Wright, G.A., Camic, C.L., Kovacs, A.J., Hegge, J.M., \& Brice, G.A. (2015). Development of an anaerobic sprint running test using a nonmotorized treadmill. Journal of Strength and Conditioning Research, 29(8), 2197-2204. doi: 10.1519/JSC.0000000000000854.

Obermaier, K., Schmelzeisen-Redeker, G., Schoemaker, M., Klötzer, H.M., Kirchsteiger, H., Eikmeier, H., et al. (2013). Performance evaluations of continuous glucose monitoring systems: Precision absolute relative deviation is part of the assessment. Journal of Diabetes Science and Technology, 7(4), 824-832. doi: 10.1177/193229681300700404

Robinson, C.S., \& Sharp, P. (2012). Tighter accuracy standards within point-of-care blood glucose monitoring: How six commonly used systems compare. Journal of Diabetes Science and Technology, 6(3), 547-554. doi: $10.1177 / 193229681200600309$

Suh, S., Paik, I., \& Jacobs, K.A. (2007). Regulation of blood glucose homeostasis during prolonged exercise. Molecules and Cells, 23(3), 272-279.

Tack, C., Pohlmeier, H., Behnke, T., Schmid, V., Grenningloh, M., Forst, T., et al. (2012). Accuracy evaluation of five blood glucose monitoring systems obtained from the pharmacy: A European multicenter study with 453 subjects. Diabetes Technology and Therapeutics, 14(4), 330-337. doi: 10.1089/dia.2011.0170

Thomas, L.E., Kane, M.P., Bakst, G., Busch, R.S., Hamilton, R.A., \& Abelseth, J.M. (2008). A glucose meter accuracy and precision comparison: The FreeStyle Flash versus the Accu-Check Advantage, Accu-Check Compact Plus, Ascenia Contour, and the BD Logic. Diabetes Technology and Therapeutics, 10(2), 102-110. doi: 10.1089/ dia.2007.0244

Vincent, J., \& Weir, J.P. (2012). Statistics in kinesiology ( $4^{\text {th }}$ ed.). Champaign, IL: Human Kinetics.

YSI Life Sciences. (2009). User's Manual YSI 2300 STAT PLUS Glucose and L-Lactate Analyzer. Retrieved January 4, 2018 from: https://www.ysi.com/File\%20Library/Documents/Manuals\%20for\%20Discontinued\%20Products/ YSI-2300-Stat-Plus-manual-j.pdf

Zinker, B.A., Britz, K., \& Brooks, G.A. (1990). Effects of a 36-hour fast on human endurance and substrate utilization. Journal of Applied Physiology, 69(5), 1849-1855. doi: 10.1152/jappl.1990.69.5.1849.

Submitted: November 6, 2018

Accepted: January 8, 2019.

Published Online First: November 7, 2019
Correspondence to:

Assoc. Prof. Clayton L. Camic, Ph.D.

Kinesiology and Physical Education

228 Anderson Hall

Northern Illinois University

DeKalb, Illinois, USA 60115

Phone: (815) 753-3415

Fax: (815)753-1413

E-mail: ccamic1@niu.edu 